\title{
ENSINO E PESQUISA NOS ESTADOS UNIDOS: ALGUMAS CARACTERÍSTICAS DOS PRINCIPAIS PROGRAMAS DE DOUTORADO EM CONTABILIDADE
}

\author{
EDUCATION AND RESEARCH IN THE UNITED STATES: \\ SOME CHARACTERISTICS OF THE MAJOR ACCOUNTING PH.D. PROGRAMS
}

\author{
FERNANDO DAL-RI MURCIA \\ Doutorando em Controladoria e Contabilidade \\ pela Faculdade de Economia, Administração e Contabilidade \\ da Universidade de São Paulo - Campus Capital \\ E-mail: fernandomurcia@hotmail.com
}

\author{
JOSÉ ALONSO BORBA \\ Professor Doutor do Departamento de Ciências Contábeis \\ do Centro Sócio-Econômico \\ da Universidade Federal de Santa Catarina - Campus Trindade \\ E-mail:jalonso@cse.ufsc.br
}

\author{
GRACIELI AMBRÓSIO \\ Mestranda em Contabilidade \\ pelo Centro Sócio-Econômico \\ da Universidade Federal de Santa Catarina - Campus Trindade \\ E-mail:newgraci@yahoo.com.br
}

\section{RESUMO}

O presente trabalho busca conhecer algumas características dos principais programas de doutorado em contabilidade dos Estados Unidos. Para a seleção da amostra deste estudo, utilizou-se, como base, o ranking divulgado pela revista U.S. News \& World Report-America's Best Graduate Schools in Accounting-2006. Esse ranking lista os 31 principais programas de pós-graduação em contabilidade nos Estados Unidos. Contudo, para o presente trabalho, 3 dessas universidades foram excluídas, restando para análise um total de 28 universidades. A coleta dos dados foi realizada nos sites eletrônicos dos programas selecionados. Buscou-se identificar 4 características desses programas de doutorado: critérios do processo seletivo, exigências para a obtenção do título de Ph.D. in Accounting, disciplinas oferecidas e perfil do corpo docente. Os resultados encontrados evidenciam algumas características dos programas analisados que podem permitir um benchmarking, possibilitando um crescimento da pesquisa acadêmica brasileira. Nesse sentido, o trabalho contribui para a construção e o aperfeiçoamento da contabilidade, à medida que evidencia possibilidades de melhorias nos programas de pós-graduação no Brasil.

Palavras-chave: Doutorado em Contabilidade; Programas de Pós-Graduação; Ensino e Pesquisa nos Estados Unidos.

\section{ABSTRACT}

This paper explores some characteristics of the best Accounting Ph.D. programs in the United States. The sample selection was based on the ranking published by the U.S. News \& World Report - America's Best Graduate Schools in Accounting-2006. This publication lists the best 31 graduate programs in accounting in the United States. However, for this study, 3 schools have been excluded, leaving a total of 28 Universities for analysis. The data has been collected throughout the university's websites. The characteristics analyzed were: admission requests, degree requirements, disciplines offered and faculty profile. This research aims at contributing to the Brazilian literature throughout a benchmarking in the American Ph.D. programs. In this sense, this work enables some possibilities for improvement in the Brazilian graduate programs.

Keywords: Ph.D. Programs in Accounting; Graduate Programs; Education and Research in North-American Universities. 


\section{INTRODUÇÃO}

O primeiro programa de doutorado (Ph.D.) em Contabilidade nos Estados Unidos foi criado pela University of Illinois at Urbana - Champaign no ano de 1938. Liderado por Ananias C. Littleton, esse programa teve como objetivo principal refletir uma nova visão no ensino da contabilidade (BEDFORD, 1997). Com o passar dos anos, a pesquisa acadêmica em contabilidade ganhou relevância principalmente com a expansão das grandes corporações e do mercado de capitais americano. Ao contrário do Brasil, que possui apenas dois programas de doutorado em contabilidade (Universidade de São Paulo, criado em 1978, Universidade de Brasília, criado em 2006), nos Estados Unidos existem aproximadamente 82 Universidades que oferecem programas de doutorado em contabilidade ou doutorado em administração, com concentração em contabilidade (HASSELBACK, 2001).

Durante os anos de 1994-1998, os programas de doutorado em contabilidade dos Estados Unidos formaram um total de 777 doutores. Mais de $80 \%$ desses novos doutores foram formados pelos 49 maiores programas, o que demonstra uma produção concentrada (DOOGAR, 2003). Entretanto, o número de doutores formados pelos programas americanos tem decaído com o passar dos anos. Segundo os resultados de uma pesquisa realizada por Carpenter e Robson (2004), a partir de meados dos anos 90 , houve uma queda de aproximadamente $50 \%$ no número de doutores formados em contabilidade nos Estados Unidos. Para Shipley e Engle (1982), a academia possui um problema de marketing, pois ela não é atrativa o bastante para recrutar os talentos que estão no mercado de trabalho. Para Gabbin (2002), a cultura acadêmica não provê os incentivos financeiros nem tampouco a estrutura administrativa necessária para reter os melhores profissionais.

Aparentemente, o custo de oportunidade para se cursar um doutorado é outro fator que tem contribuído para o declínio na formação desses profissionais. O tempo para a obtenção do título de doutor em contabilidade (média de
3-6 anos) e a exigência de dedicação integral são alguns dos fatores que afastam profissionais dos programas de doutorado. Segundo Meyer e Titard (2000), para um profissional que possui um emprego estável desistir de seu trabalho para ingressar em um programa de doutorado é um grande sacrifício financeiro.

Em contrapartida, a demanda por doutores em contabilidade nos Estados Unidos é crescente. O trabalho de Carpenter e Robson (2004) evidenciou, através de dados coletados no site da American Accounting Association's ( $A A A)$, uma crescente disparidade entre o número de currículos de doutores que buscavam trabalho (oferta) e o número de posições em aberto (demanda). O Quadro 1 apresenta esses dados.

O Quadro 10 evidencia que a partir do ano de 1997, existe uma crescente demanda (posições em aberto) por doutores em contabilidade nos Estados Unidos que supera a oferta (currículos). Uma das razões para o aumento dessa demanda é o grande número de escolas de negócios que tem buscado afiliar-se a Association to Advance Collegiate Schools of Business - AACSB, instituição que exige um corpo de docentes qualificado (CARPENTER e ROBSON, 2004).

Visando explorar um tema ainda pouco conhecido no âmbito nacional, esta pesquisa busca identificar 4 características dos programas de doutorado em contabilidade nos Estados Unidos: critérios do processo seletivo, exigências para a obtenção do título de Ph.D. in Accounting, disciplinas oferecidas e perfil do corpo docente.

$O$ restante deste trabalho segue a seguinte ordem: Seção 2, apresenta algumas considerações sobre o Ph.D. in Accounting; Seção 3, evidencia algumas das principais pesquisas envolvendo ensino e pesquisa na área contábil; Seção 4, descreve a metodologia utilizada na pesquisa; Seção 5, ilustra os resultados e a Seção 6, traz as conclusões e recomendações deste trabalho.

\begin{tabular}{|c|c|c|}
\hline Ano & Currículos & Posições em aberto \\
\hline 1992 & 180 & 110 \\
\hline 1993 & 154 & 109 \\
\hline 1994 & 129 & 115 \\
\hline 1995 & 143 & 110 \\
\hline 1996 & 134 & 108 \\
\hline 1997 & 117 & 120 \\
\hline 1998 & 92 & 145 \\
\hline 1999 & 76 & 152 \\
\hline 2000 & 70 & 175 \\
\hline 2001 & 66 & 153 \\
\hline
\end{tabular}

Fonte: Carpenter e Robson (2004) 


\section{PH.D. IN ACCOUNTING}

O título de Ph.D, ou Philosophy Doctor, nos países de língua inglesa corresponde ao título de Doutor no Brasil. Assim, como o doutorado no cenário nacional, o Philosophy Doctor é oferecido em diversas áreas do conhecimento científico (biológicas, humanas etc.). Nos Estados Unidos (alvo de análise de estudo), na área específica de negócios, existem dois programas de doutorado: o Ph.D (Accounting, Finance, Economics etc.) e o DBA ou Doctor in Business Administration, que corresponderia ao Doutorado em Administração de Empresas no Brasil. O DBA oferece concentração nas mesmas áreas de negócios que o Ph.D (Accounting, Finance, Economics etc.) e, nesse sentido, são programas semelhantes. Durante o transcorrer deste texto, a pesquisa referir-se-á aos programas de doutorado em contabilidade nos Estados Unidos, mas em algumas universidades, como a Harvard University, por exemplo, possuem apenas o DBA com concentração em contabilidade.

Mais do que uma especialização na área de negócios, os programas de Ph.D e DBA nos Estados Unidos têm como principal objetivo formar um profissional que atuará na área de pesquisa e ensino. Esse enfoque é percebido devido ao baixo número de disciplinas específicas oferecidas nesses programas. Segundo Kinney (2003), que analisou 75 programas de doutorado na área, as disciplinas de contabilidade correspondem a apenas $21.4 \%$ do total do curso e o restante são disciplinas de metodologia, sociologia e métodos quantitativos.

Nessa mesma linha de pensamento, alguns pesquisadores como Mintzberg (2006) têm desenvolvido trabalhos no intuito de evidenciar que disciplinas como psicologia, sociologia, economia, matemática, antropologia e história são a base para a formação do gestor e do pesquisador da área de negócios. A Figura 10 ilustra o Modelo GSIA, elaborado por Mintzberg.

Segundo Mintzberg (2006), as habilidades de pensamento crítico dos estudantes das escolas de negócios não são desenvolvidas apenas por disciplinas específicas, pois as funções empresariais (Contabilidade, Marketing, TI, Estratégia etc.) são alimentadas pelas raízes, ou seja, pelas disciplinas básicas.

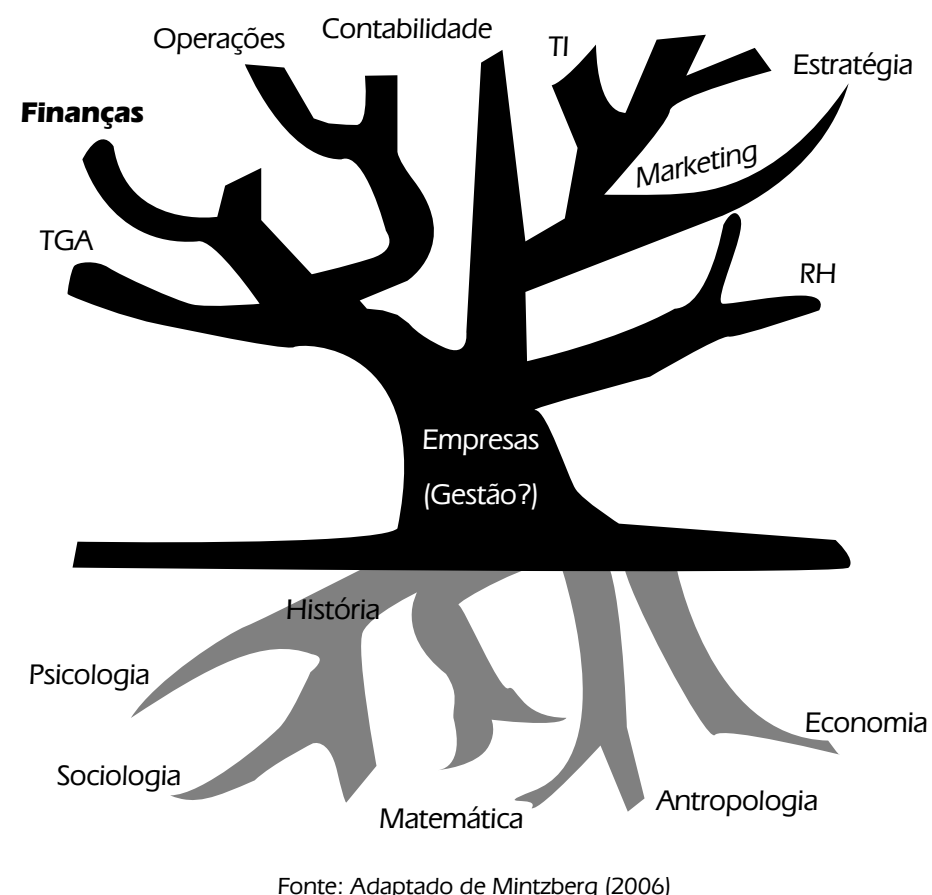

Figura 1 - Modelo GSIA de Currículo para Escolas de Negócios

\section{REVISÃO DE LITERATURA}

No âmbito nacional, nota-se a existência de poucos estudos sobre programas de pós-graduação em contabilidade. Germano (1988) desenvolveu um estudo sobre as teses de doutorado em contabilidade na Universidade de São Paulo até o ano de 1988. Marion (1992) analisou diversos aspectos do ensino da contabilidade nos Estados Unidos. Riccio, Carastan e Sakata
(1999) analisaram as características da produção acadêmica no Brasil - dissertações de mestrado e teses de doutorado - no período de 1962-1999. Contudo, no âmbito internacional, diversos acadêmicos têm desenvolvido trabalhos buscando explorar características dos programas de pós-graduação em contabilidade. O Quadro 20 ilustra algumas dessas pesquisas. 


\begin{tabular}{|c|c|}
\hline Autor/ Periódico & Pesquisa \\
\hline $\begin{array}{l}\text { Crum (1974) } \\
\text { Journal of Accountancy }\end{array}$ & $\begin{array}{l}\text { Analisou os } 63 \text { programas de doutorado em contabilidade existentes, utilizando como } \\
\text { metodologia para coleta de dados um questionário com questões sobre o programa, } \\
\text { corpo discente e corpo docente. }\end{array}$ \\
\hline $\begin{array}{l}\text { Shipley e Engle (1982) } \\
\text { Management Accounting }\end{array}$ & $\begin{array}{l}\text { Desenvolveram um trabalho teórico que buscou evidenciar a necessidade de doutores em } \\
\text { contabilidade nos Estados Unidos. }\end{array}$ \\
\hline $\begin{array}{l}\text { Johnson (1985) } \\
\text { The Accounting Review }\end{array}$ & $\begin{array}{l}\text { Avaliou as políticas e práticas educacionais dos programas de doutorado em contabilida- } \\
\text { de nos Estados Unidos. }\end{array}$ \\
\hline $\begin{array}{l}\text { Bell, Frecka e Solomon (1993) } \\
\text { Accounting Horizons }\end{array}$ & $\begin{array}{l}\text { Realizaram uma pesquisa empírica em uma amostra de } 473 \text { professores de } 31 \text { universida- } \\
\text { des americanas testando a relação entre a produção científica e a eficiência no ensino. }\end{array}$ \\
\hline $\begin{array}{l}\text { Carcacello et al. (1994) } \\
\text { Issues in Accounting Education }\end{array}$ & $\begin{array}{l}\text { Analisaram os custos dos programas de doutorado em contabilidade nos Estados Unidos } \\
\text { e o apoio financeiro (bolsa de estudos, teaching assistance etc.) oferecido pelas Universi- } \\
\text { dades aos estudantes. }\end{array}$ \\
\hline $\begin{array}{l}\text { Reinstein e Hasselback (1998) } \\
\text { Journal of Accounting Education }\end{array}$ & $\begin{array}{l}\text { Desenvolveram um artigo que revisou a literatura existente sobre a produção científica } \\
\text { dos professores de contabilidade nos Estados Unidos e estabeleceram um modelo para a } \\
\text { avaliação da produtividade desses docentes. }\end{array}$ \\
\hline $\begin{array}{l}\text { Stammerjohan e Hall (2002) } \\
\text { Journal of Accounting Education }\end{array}$ & $\begin{array}{l}\text { Avaliaram e estabeleceram um ranking para os programas de doutorado em contabilida- } \\
\text { de nos Estados Unidos com base em quesitos, como: produção acadêmica, colocação dos } \\
\text { doutores formados no programa, departamento de contabilidade, entre outros. }\end{array}$ \\
\hline $\begin{array}{l}\text { Beattie e Goodacre (2003) } \\
\text { The British Accounting Review }\end{array}$ & $\begin{array}{l}\text { Realizaram um estudo no Reino Unido sobre os padrões de publicação da comunidade } \\
\text { acadêmica das áreas de contabilidade/finanças. }\end{array}$ \\
\hline $\begin{array}{l}\text { Brown e Laksmana (2003) } \\
\text { Social Science Research Network }\end{array}$ & $\begin{array}{l}\text { Estabeleceram um ranking para os programas de doutorado em contabilidade nos Esta- } \\
\text { dos Unidos com base no número de downloads dos artigos publicados no Social Science } \\
\text { Research Network (SSRN). }\end{array}$ \\
\hline $\begin{array}{l}\text { Doogar (2003) } \\
\text { Advances in Accounting Education }\end{array}$ & $\begin{array}{l}\text { Analisou a oferta e a demanda dos doutorados em contabilidade nos Estados Unidos e } \\
\text { sugeriu um modelo que leva em conta critérios financeiros na avaliação dos docentes } \\
\text { vinculados aos programas de doutorado em contabilidade. }\end{array}$ \\
\hline $\begin{array}{l}\text { Everett, Klamm e Stoltzfus (2004) } \\
\text { Journal of Accounting Education }\end{array}$ & $\begin{array}{l}\text { Sumarizaram e analisaram a produtividade acadêmica de } 87 \text { programas de doutorado em } \\
\text { contabilidade no período de } 1992-1996 \text {. }\end{array}$ \\
\hline
\end{tabular}

Fonte: Elaborado pelos autores

Quadro 2 Estudos envolvendo Doutorados em Contabilidade

\section{METODOLOGIA}

Para a seleção das Universidades Americanas, utilizouse como base o ranking divulgado pela revista U.S. News E World Report - America's Best Graduate Schools in Accounting 2006. Essa revista de negócios utiliza diversos critérios na elaboração do ranking como, por exemplo, o índice de aceitação do programa, o índice professor/aluno, a avaliação dos outros programas (peer review), valor gasto em pesquisa nos anos anteriores e número de doutores formados pelo programa.

Nesse ranking, estão listados os 31 melhores programas de pós-graduação em contabilidade (mestrado e doutorado) nos Estados Unidos. Para o presente estudo, foram excluídas três Universidades: University of Virginia, Brigham Young University e University of Notre Dame, pois não possuíam um programa de doutorado em contabilidade ou um programa de administração com concentração em contabilidade. (Essas três universidades possuem apenas programas de mestrado em contabilidade). O Quadro $3 \bullet$ apresenta os programas selecionados para este estudo juntamente com o ranking da U.S. News E World Report America's Best Gra- duate Schools para as áreas de contabilidade, finanças, administração e negócios.

O Quadro 3 evidencia que os principais programas de pós-graduação na área de contabilidade também estão bem colocados nas áreas de finanças, administração e negócios. Contudo, a Harvard University, por exemplo, apesar de ser a primeira colocada na área de negócios, é apenas a décima segunda $\left(12^{\mathrm{a}}\right)$ na área de contabilidade e a primeira nessa área é a University of Pennsylvania.

Apesar de não levar em conta diversos critérios acadêmicos como publicação em periódicos e endogenia do corpo docente, os rankings elaborados por revistas de negócios (U.S. News E World Report, Times, Business Week etc.) são considerados importantes, pois influenciam no recrutamento de estudantes. Segundo Siemens et al. (2005), grande parte dos estudantes utiliza os rankings publicados pelas revistas de negócios na escolha de universidades e cursos. Em alguns casos, programas de pós-graduação em negócios têm um aumento de até $40 \%$ no número de novos estudantes quando melhoram a suas performances nos rankings (WILLIAMS, 2001). 


\begin{tabular}{|c|c|c|c|c|}
\hline \multirow{2}{*}{ Universities } & \multicolumn{4}{|c|}{ U. S. News \& World Report } \\
\hline & Accounting & Management & Finance & Business \\
\hline University of Pennsylvania & 1 & 5 & 1 & 2 \\
\hline University of Chicago & 2 & 13 & 2 & 8 \\
\hline University of Texas-Austin & 3 & 19 & 18 & 18 \\
\hline University of Illinois-Urbana-Champaign & 4 & 27 & 25 & 27 \\
\hline University of Michigan-Ann Arbor & 5 & 3 & 11 & 10 \\
\hline Stanford University (CA) & 6 & 4 & 4 & 2 \\
\hline New York University & 7 & 12 & 3 & 13 \\
\hline University of Southern California & 8 & 20 & 26 & 26 \\
\hline University of North California-Chapel Hill & 9 & 18 & - & 21 \\
\hline Northwestern University & 10 & 2 & 8 & 4 \\
\hline Columbia University (NY) & 11 & 8 & 5 & 9 \\
\hline Harvard University (MA) & 12 & 1 & 7 & 1 \\
\hline Indiana University-Bloomington & 13 & 16 & 19 & 27 \\
\hline Ohio State University & 14 & 26 & 16 & 21 \\
\hline University of California-Berkeley & 15 & 9 & 10 & 6 \\
\hline Cornell University (NY) & 16 & - & 15 & 15 \\
\hline University of Rochester (NY) & 17 & - & 12 & 23 \\
\hline Massachusetts Institute of Technology & 18 & 11 & 6 & 4 \\
\hline University of Florida & 19 & 29 & 21 & 42 \\
\hline University of Washington & 20 & - & - & 18 \\
\hline University of Wisconsin-Madison & 21 & - & 22 & 37 \\
\hline Texas A\&M University-College Station & 22 & - & - & 32 \\
\hline University of Georgia & 23 & - & - & 45 \\
\hline Arizona State University-Main Campus & 24 & - & - & 31 \\
\hline University of Arizona & 25 & - & - & 40 \\
\hline University of lowa & 26 & - & - & 37 \\
\hline Michigan State University & 27 & - & - & 32 \\
\hline University of California Los Angeles & 28 & - & 9 & 11 \\
\hline
\end{tabular}

Fonte: Elaborado pelos autores

Quadro 3 ॥ Ranking dos programas de pós-graduação em Contabilidade

Os rankings de revistas de negócios, também, funcionam como uma forma de auto-avaliação para os programas de pós-graduação, pois ilustram pontos que podem ser melhorados. Segundo Policano (2005), esses rankings fornecem às escolas um feedback externo que evidencia 0 que administradores, acadêmicos e recrutadores pensam a respeito de um determinado programa.

Embora a amostra deste estudo seja composta por apenas 28 programas de doutorado do total de 82 existentes nos Estados Unidos, os profissionais formados pelos programas selecionados, até março de 2005 , representam aproximadamente $47 \%$ do total de 6472 doutores em contabilidade formados pelas universidades desse país (HASSELBACK, 2005). O Quadro 40 evidencia os doutores formados pelas universidades durante os anos anteriores a 2001, e nos anos de 2001, 2002, 2003, 2004, e meados de 2005.

Percebe-se que as universidades americanas selecionadas formam um número relativamente baixo de doutores em contabilidade por ano. Das universidades listadas no Quadro 4, apenas a Universidade of Texas-Austin, a University of Illinois, a University of Indiana, a University of Wisconsin e a Michigan State University formaram mais doutores em contabilidade que a Universidade de São Paulo, por exemplo, que formou aproximadamente 150 doutores nessa área.

Após a seleção dos 28 programas de doutorado, procurou-se coletar os dados através de buscas nos sites eletrônicos das universidades. Algumas delas possuem um site eletrônico específico para o programa de doutorado, enquanto outras possuem um site do departamento de contabilidade que contém as informações sobre os programas de doutorado. A coleta de dados foi realizada entre os dias 5 de agosto de 2005 e 18 de junho de 2006 e buscou-se identificar 4 características:

- exigências de entrada no programa: características do processo seletivo dos programas de doutorados;

- requisitos para obtenção do título de doutor: tese, provas e publicações;

- disciplinas: como é composto o currículo do programa de doutorado em contabilidade;

- corpo docente: total de professores e índice de endogenia dos programas. 


\begin{tabular}{|c|c|c|c|c|c|c|c|}
\hline Universities & Pre-2001 & 2001 & 2002 & 2003 & 2004 & 2005 & Total \\
\hline Pennsylvania & 26 & 0 & 1 & 0 & 2 & 2 & 31 \\
\hline Chicago & 72 & 0 & 0 & 0 & 1 & 0 & 73 \\
\hline Texas-Austin & 247 & 3 & 2 & 0 & 4 & 2 & 258 \\
\hline Illinois & 329 & 4 & 2 & 4 & 0 & 0 & 339 \\
\hline Michigan & 118 & 0 & 1 & 0 & 0 & 0 & 119 \\
\hline Stanford & 72 & 0 & 0 & 0 & 0 & 0 & 72 \\
\hline New York & 85 & 4 & 1 & 4 & 4 & 1 & 99 \\
\hline Southern California & 97 & 0 & 1 & 0 & 2 & 0 & 100 \\
\hline North Carolina & 71 & 1 & 1 & 2 & 1 & 0 & 76 \\
\hline Northwestern & 63 & 2 & 1 & 0 & 3 & 0 & 69 \\
\hline Columbia & 71 & 3 & 1 & 1 & 2 & 1 & 79 \\
\hline Harvard & 103 & 1 & 1 & 0 & 1 & 0 & 106 \\
\hline Indiana & 146 & 2 & 1 & 1 & 0 & 0 & 150 \\
\hline Ohio State & 126 & 2 & 4 & 1 & 0 & 0 & 133 \\
\hline Ca-Berkeley & 96 & 1 & 0 & 1 & 0 & 0 & 98 \\
\hline Cornell & 42 & 0 & 2 & 1 & 4 & 0 & 49 \\
\hline Rochester & 25 & 0 & 2 & 0 & 0 & 0 & 27 \\
\hline MIT & 10 & 0 & 1 & 0 & 0 & 0 & 11 \\
\hline Florida & 108 & 1 & 0 & 0 & 2 & 0 & 111 \\
\hline Washington & 118 & 3 & 2 & 3 & 1 & 0 & 127 \\
\hline Wisconsin & 140 & 2 & 2 & 2 & 4 & 3 & 153 \\
\hline Texas A\&M & 121 & 3 & 3 & 2 & 6 & 1 & 136 \\
\hline Georgia & 105 & 3 & 1 & 1 & 1 & 0 & 111 \\
\hline Arizona State & 95 & 2 & 3 & 1 & 1 & 0 & 102 \\
\hline Arizona & 70 & 7 & 1 & 3 & 3 & 0 & 84 \\
\hline lowa & 59 & 2 & 3 & 0 & 1 & 1 & 66 \\
\hline Michigan State & 176 & 1 & 3 & 0 & 4 & 0 & 184 \\
\hline UCLA & 59 & 0 & 0 & 0 & 0 & 0 & 59 \\
\hline TOTAL & 2850 & 47 & 40 & 27 & 47 & 11 & 3022 \\
\hline
\end{tabular}

Fonte: Adaptado de Hasselback (2005)

Quadro 4 Doutores formados nas universidades selecionadas

\section{ANÁLISE DOS RESULTADOS}

A apresentação dos resultados desta pesquisa está dividida em 4 partes. A primeira parte evidencia os critérios do processo seletivo dos programas analisados. Posteriormente, são analisados os requerimentos para a obtenção do título de Ph.D in Accounting. A terceira parte apresenta uma breve análise das disciplinas oferecidas nesses programas e a última evidencia algumas considerações a respeito do corpo docente vinculado às universidades analisadas.

\subsection{O Processo Seletivo}

Basicamente, os programas de doutorado em contabilidade analisados possuem 6 critérios de seleção: GMAT/ GRE, TOEFL, cartas de recomendação, GPA, entrevista e dedicação exclusiva. $O$ Quadro $5 \bullet$ ilustra os resultados encontrados.

\subsubsection{GMAT / GRE}

Todos os programas analisados exigem o General Management Admission Test (GMAT) ou o Graduate Record Exa- mination (GRE) em seu processo seletivo. Esses testes não exigem um conhecimento contábil específico. Pelo contrário, são testes que analisam o conhecimento quantitativo, analítico e verbal dos candidatos. O GMAT e o GRE poderiam ser comparados ao teste da ANPAD que é exigido pela grande maioria dos programas de mestrado e doutorado na área de administração e contabilidade no Brasil. A pontuação exigida pelos 28 programas dos Estados Unidos varia entre 600 e 740 pontos e uma grande parte das universidades não exige uma pontuação mínima.

\subsubsection{TOEFL}

O Test of English as a Foreign Language -TOEFL como o próprio nome já diz, é um teste para indivíduos que não tem o Inglês como língua materna. Assim como o GMAT/GRE, o TOELF é um requerimento do processo seletivo da maioria programas de doutorado em contabilidade analisados. $A$ pontuação exigida por esses programas varia de 550- 650 alguns deles não exigem uma pontuação mínima. 


\begin{tabular}{|c|c|c|c|c|c|c|}
\hline Universities & GMAT/GRE & TOEFL & Cartas & G.P.A & D.E. & Entrevista \\
\hline Pennsylvania & s.p.m & s.p.m & 3 & 3.0 & $\operatorname{sim}$ & não \\
\hline Chicago & s.p.m & 600 & 2 & n.m & $\operatorname{sim}$ & não \\
\hline Texas-Austin & 700 & s.p.m & 3 & 3.0 & $\operatorname{sim}$ & não \\
\hline Illinois & 700 & 600 & 3 & 3.0 & $\operatorname{sim}$ & não \\
\hline Michigan & s.p.m & 600 & 3 & n.m & $\operatorname{sim}$ & não \\
\hline Stanford & s.p.m & 600 & 1 & s.p.m & $\operatorname{sim}$ & não \\
\hline New York & s.p.m & s.p.m & 2 & s.p.m & $\operatorname{sim}$ & não \\
\hline Southern California & 700 & 650 & 3 & n.m & $\operatorname{sim}$ & $\operatorname{sim}$ \\
\hline North Carolina & 720 & s.p.m & 3 & n.m & $\operatorname{sim}$ & não \\
\hline Northwestern & s.p.m & 600 & 2 & 3.0 & $\operatorname{sim}$ & não \\
\hline Columbia & 730 & s.p.m & 2 & n.m & $\operatorname{sim}$ & não \\
\hline Harvard & $90 \%$ & 600 & 3 & n.m & $\operatorname{sim}$ & não \\
\hline Indiana & 700 & s.p.m & 3 & n.m & $\operatorname{sim}$ & $\operatorname{sim}$ \\
\hline Ohio State & 600 & s.p.m & 3 & n.m & $\operatorname{sim}$ & $\operatorname{sim}$ \\
\hline Ca-Berkeley & s.p.m & 570 & 3 & 3.0 & $\operatorname{sim}$ & não \\
\hline Cornell & 720 & 600 & 2 & n.m & $\operatorname{sim}$ & $\operatorname{sim}$ \\
\hline Rochester & 680 & s.p.m & 2 & n.m & $\operatorname{sim}$ & não \\
\hline MIT & s.p.m & 577 & 2 & n.m & $\operatorname{sim}$ & não \\
\hline Florida & 690 & 550 & 2 & 3.0 & $\operatorname{sim}$ & não \\
\hline Washington & 700 & s.p.m & 3 & n.m & $\operatorname{sim}$ & não \\
\hline Wisconsin & $90 \%$ & 600 & 3 & 3.0 & $\operatorname{sim}$ & não \\
\hline Texas A\&M & 690 & 550 & 3 & n.m & $\operatorname{sim}$ & $\operatorname{sim}$ \\
\hline Georgia & 700 & s.p.m & 3 & 3.0 & $\operatorname{sim}$ & não \\
\hline Arizona State & s.p.m & 550 & 3 & 3.0 & $\operatorname{sim}$ & não \\
\hline Arizona & 620 & 600 & 3 & 3.0 & $\operatorname{sim}$ & não \\
\hline lowa & 710 & 630 & 3 & 3.0 & $\operatorname{sim}$ & não \\
\hline Michigan State & 600 & 600 & 3 & 3.0 & $\operatorname{sim}$ & $\operatorname{sim}$ \\
\hline UCLA & 740 & 560 & 3 & 3.5 & $\operatorname{sim}$ & não \\
\hline
\end{tabular}

Fonte: Elaborado pelos autores

Notas: * s.p.m. = sem pontuação mínima

** n.m. = não mencionado.

Quadro 5 Critérios do Processo Seletivo

\subsubsection{Cartas de Recomendação (C.R.)}

Todos os programas analisados exigem cartas de recomendação no processo seletivo do doutorado em contabilidade. O número de cartas de recomendação varia de 1 a 3. Essas cartas podem ser originárias de outros professores universitários ou de profissionais do mercado de trabalho.

\subsubsection{Grade Point Average (G.P.A.)}

Todas as 28 universidades selecionadas nesse estudo analisam o currículo do candidato no processo seletivo. Como os estudantes americanos dificilmente publicam artigos científicos antes do Doutorado (a graduação não exige a monografia para a conclusão de curso e a grande maioria dos mestrados americanos não exige a dissertação de mestrado), o foco da análise de currículo é o Grade Point Average (GPA). Essa é uma média das notas dos estudantes durante os cursos de graduação e pós-graduação que varia entre 0-4. O GPA mínimo exigido pela maioria dos programas de doutorado é 3.0 (equivale no Brasil a uma média geral B), mas a University of California at Los Angeles (UCLA), por exemplo, exige 3.5.

\subsubsection{Dedicação Exclusiva (D.E.)}

Todos os 28 programas de doutorado analisados exigem dedicação exclusiva (full-time). Alguns deles exigem essa dedicação apenas nos dois primeiros anos de estudo, enquanto o estudante cursa as disciplinas. Entretanto, a grande parte dos programas exige dedicação exclusiva até que o estudante defenda sua tese de doutorado. Em contrapartida, a maioria das universidades americanas analisadas oferece bolsa total de estudos para os alunos de doutorado em contabilidade. Essas Universidades, também, oferecem outras formas de apoio financeiro a seus estudantes de doutorado. A University of Texas - Austin, por exemplo, oferece o cargo de Teaching Assistant - TA como forma de ajuda financeira aos seus estudantes de doutorado. A Columbia University oferece, além de bolsa de estudos e do TA, um apoio financeiro na elaboração da tese de doutorado. 


\subsubsection{Entrevista}

Apenas 6 das 28 universidades analisadas entrevistam seus candidatos na seleção de seus programas de doutorado em contabilidade. Como os programas de doutorado analisados possuem candidatos de diversas partes do mundo, deduz-se que a exigência da entrevista resultaria em um empecilho muito grande, que a maioria das universidades americanas prefere não causar, temendo pela perda de bons candidatos estrangeiros. Entretanto, na seleção de bolsas de estudo e nos outros tipos de programas de apoio financeiro a entrevista é exigida.

\subsubsection{As Não-Exigências}

Alguns dos critérios utilizados no processo seletivo do programa de doutorado em contabilidade da USP não são exigidos por nenhuma das 28 Universidades Americanas analisadas, como por exemplo:

- prova específica de contabilidade;

- análise de pré-projeto de dissertação/ tese;

- diploma de mestrado;

- publicações científicas.

\subsection{Requerimentos de Saída - Obtenção do título de Ph.D in Accounting}

Além da aprovação nas disciplinas do programa, do qualify e da defesa da tese de doutorado, dois são os requerimentos exigidos pelas Universidades Americanas para a obtenção do título de Ph.D in Accounting: exame de suficiência e publicações científicas.

\subsubsection{Exames de Suficiência}

Todos os programas analisados exigem a aprovação em um exame de suficiência. Após o término das disciplinas do programa de doutorado, os estudantes devem comprovar seus conhecimentos através de uma prova que engloba disciplinas como: contabilidade, estatística/econometria e metodologia. Somente após a aprovação nesse exame é que os estudantes se tornam Ph.D Candidates (candidatos ao título de Doutor). Caso sejam reprovados no exame, os estudantes possuem mais uma chance, geralmente em um prazo de 6 meses, para estudar e realizar o exame novamente. Na University of Pennsylvania - Wharton Business School, ranqueada no primeiro lugar pela revista da U.S. News $\mathcal{E}$ World Report, o exame de suficiência é realizado na forma escrita e oral.

Percebe-se, dessa maneira, que o doutorado em contabilidade nos Estados Unidos é dividido em duas grandes partes. Nos primeiros dois anos, o foco é a preparação para o exame de suficiência. Nesse período, os estudantes cursam as disciplinas obrigatórias e eletivas nas quais são cobradas provas. A partir do terceiro ano, quando o estudante se torna um Ph.D Candidate o foco é a pesquisa, a elaboração do projeto, a defesa do Qualify e, finalmente, a defesa da Tese de Doutorado.

\subsubsection{Publicações Acadêmicas}

A publicação acadêmica, também, é uma das exigências para a obtenção do título de Ph.D in Accounting. A grande maioria dos programas exige entre uma e duas publicações dos alunos de doutorado. O Massachusetts Institute of Technology (MIT) é o único programa de doutorado analisado que exige 3 publicações acadêmicas. Entretanto, vale ressaltar que os programas analisados não exigem publicações em periódicos conceituados de contabilidade como, por exemplo, na Accounting Review (TAR), na Accounting, Organization and Society (AOS), no Journal of Accouting Research (JAR) ou no Journal of Accounting and Economics (JAE). Provavelmente, devido à dificuldade de publicação nesses periódicos, os programas exigem, apenas, a elaboração de working papers, que são geralmente enviados para congressos no intuito de receber críticas construtivas e contribuições que ajudarão na elaboração da Tese de Doutorado.

Percebe-se, assim, que o foco dos programas de doutorado em contabilidade nos Estados Unidos não é a publicação e sim a formação do pesquisador, pois durante todo o período de doutorado (que em alguns casos leva de 5, 6 ou mais anos) são exigidos apenas dois artigos acadêmicos publicados em congressos ou accounting foruns.

\subsection{Disciplinas}

Nessa análise, os resultados evidenciaram que número total de disciplinas exigidas, bem como o número de disciplinas obrigatórias para a obtenção do titulo de Ph.D in Accounting, varia dependendo do programa. O Quadro 60 mostra esses resultados.

Do mesmo modo, as análises evidenciaram que os programas analisados possuem um número relativamente alto de disciplinas envolvendo métodos quantitativos. Disciplinas como economia, estatística e econometria fazem parte do currículo na grande maioria dos cursos analisados. Percebem-se, também, a preocupação desses programas com as disciplinas de metodologia, sociologia e psicologia.

Buscou-se verificar, igualmente, as disciplinas obrigatórias do programa de doutorado em contabilidade da Universidade de São Paulo. O Quadro $7 \bullet$ apresenta as disciplinas obrigatórias do programa da USP e as disciplinas obrigatórias dos 4 primeiros programas americanos de acordo com o ranking da U.S. News \& World Report America's Best Graduate Schools in Accounting 2006.

Tanto as disciplinas dos programas de doutorado norte-americanos analisados quanto as disciplinas do programa de doutorado da USP possuem uma média de 4 horasaula semanais, e têm duração de 1 semestre. Contudo, as exigências (leituras, tarefas, artigo acadêmico, apresentações etc.) de determinadas disciplinas podem variar. Nesse sentido, ressalte-se que, para a realização de comparações mais profundas, faz-se necessário verificar também as ementas das disciplinas, não analisadas neste estudo.

Observa-se que o foco principal dos programas de doutorado norte-americanos é a formação do Accounting Researcher (pesquisador em contabilidade). As disciplinas como estatística, metodologia científica, psicologia e sociologia são privilegiadas em detrimento de disciplinas na área especifica da contabilidade. Ressalte-se que o Quadro 


\begin{tabular}{|c|c|c|}
\hline Universidade & Total de disciplinas & Disciplinas Obrigatorias \\
\hline Pennsylvania & 20 & 5 \\
\hline Chicago & 19 & 17 \\
\hline Texas-Austin & 15 & 9 \\
\hline Illinois & 16 & 8 \\
\hline Michigan & 20 & 18 \\
\hline Stanford & 20 & 9 \\
\hline New York & 20 & 14 \\
\hline Southern California & 20 & 12 \\
\hline North Carolina & n.m & 9 \\
\hline Northwestern & 10 & 10 \\
\hline Columbia & 20 & 10 \\
\hline Harvard & 13 & 8 \\
\hline Indiana & 22 & n.m \\
\hline Ohio State & 16 & 12 \\
\hline Ca-Berkeley & 23 & 17 \\
\hline Cornell & n.m & n.m \\
\hline Rochester & 24 & 20 \\
\hline MIT & n.m & n.m \\
\hline Florida & 12 & 12 \\
\hline Washington & 22 & 17 \\
\hline Wisconsin & 12 & 11 \\
\hline Texas A\&M & 23 & 21 \\
\hline Georgia & 23 & 20 \\
\hline Arizona State & 24 & 18 \\
\hline Arizona & 30 & 18 \\
\hline lowa & 23 & 20 \\
\hline Michigan State & 22 & 16 \\
\hline UCLA & n.m & 19 \\
\hline
\end{tabular}

Fonte: Elaborado pelos autores

Nota: * n.m. = não mencionado

\section{Quadro 6 | Disciplinas Obrigatórias}

\begin{tabular}{|c|c|}
\hline Universidade & Disciplinas Obrigatórias \\
\hline USP & Contabilometria, Teoria Avançada da Contabilidade. \\
\hline Pennsylvania & $\begin{array}{l}\text { Analytical Research in Accounting, Empirical Research in Accounting, Microeconomics Theory, Game Theory and } \\
\text { Applications, Seminar in Finance. }\end{array}$ \\
\hline Chicago & $\begin{array}{l}\text { Price Theory I, Price Theory II, Microeconomics I, Microeconomics II, Macroeconomics I, Calculus I, Calculus II, } \\
\text { Calculus III, Math for Business Research Real Analysis I, Math for Business Research Real Analysis II, Math for Bu- } \\
\text { siness Research Real Analysis III, Econometrics I, Econometrics II, Econometrics III, Topics in Accounting Research, } \\
\text { Empirical Research in Accounting, Topics in Empirical Accounting Research. }\end{array}$ \\
\hline Texas-Austin & $\begin{array}{l}\text { Microeconomics, Probability, Statistics, Econometrics, Contemporary Accounting Topics, Research Methods } \\
\text { in Accounting, Empirical Research in Accounting, Analytical Research in Accounting, Behavioral Research in } \\
\text { Accounting. }\end{array}$ \\
\hline Illinois & $\begin{array}{l}\text { Economics I, Economics II, Statistics I, Research Methodology in Accounting, Contemporary Research in Accoun- } \\
\text { ting I, Contemporary Research in Accounting II, Contemporary Research in Accounting III, Research Project in } \\
\text { Accounting. }\end{array}$ \\
\hline
\end{tabular}

Fonte: Elaborado pelos autores

\section{Quadro 7 Disciplinas Obrigatórias}

7 não teve como objetivo criticar o enfoque do doutorado da USP ou o enfoque dos doutorados norte-americano.
Buscou-se, apenas, evidenciar as disciplinas obrigatórias exigidas por esses programas. 


\subsection{Perfil do Corpo Docente}

Provavelmente, a pesquisa acadêmica em contabilidade tem seu pólo principal nos Estados Unidos. Esse país é responsável por grande parte da disseminação do conhecimento contábil. Dos seis periódicos de contabilidade, avaliados quantitativamente pelo Journal of Citation Report - JCR (2004), quatro são americanos: Journal of Accounting and Economics (JAE), Journal of Accounting Research (JAR), Accounting Review (TAR) e Auditing (AJPT). Algumas das principais escolas de negócios do mundo estão localizadas em território americano. Além disso, alguns dos principais pesquisadores acadêmicos da área contábil estão vinculados aos programas de doutorado analisados nesse estudo. O Quadro 80 apresenta alguns desses pesquisadores.

Vale ressaltar que a escolha desses pesquisadores não utilizou nenhum critério cientifico especifico, sendo utilizado nessa seleção apenas o julgamento dos autores. Logo, esse quadro possuía apenas um caráter ilustrativo.

No intuito de analisar a composição do corpo docente das universidades selecionadas, procurou-se identificar o índice de endogenia dos respectivos programas. Alguns sites eletrônicos não informavam os professores que estavam associados ao programa de doutorado, apenas os professores que estavam associados ao departamento de contabilidade. Para padronizar a análise, optou-se por coletar as informações dos departamentos de contabilidade.

As análises evidenciaram um baixo grau de endogenia nas universidades analisadas. A grande maioria dos departamentos de contabilidade apresenta uma grande diversidade na composição do seu corpo docente. Esses resultados são evidenciados no Quadro $9 \boldsymbol{O}$ que apresenta os dados dos programas de doutorado analisados juntamente com os dados do programa de doutorado da USP.

A inclusão da Universidade de São Paulo nas análises objetivou ilustrar algumas características do doutorado em contabilidade brasileiro. Afinal, para analisar as características dos programas de doutorado em contabilidade americanos, fez-se necessário, primeiramente, explorar as características do programa da USP, que até a recente aprovação do programa de doutorado da UnB, era o único responsável pela formação de doutores em contabilidade no Brasil.

O Quadro 9 evidencia que os programas americanos possuem um baixo grau de endogenia. Merece destaque o fato de que, na maioria das vezes, as melhores universidades americanas não recrutam doutores formados pelos seus próprios programas, como é o caso da University of Pennsylvania que não possui nenhum egresso do programa de doutorado no seu corpo docente.

\begin{tabular}{|l|l|}
\multicolumn{1}{|c|}{ Pesquisador } & \multicolumn{1}{c|}{ Universidade } \\
Robert S. Kaplan & Harvard University \\
\hline James Ohlson & Arizona State University \\
\hline Jerold L. Zimmerman & University of Rochester \\
\hline William H. Beaver & Stanford University \\
\hline Ira Solomon & University of Illinois \\
\hline Ray Ball & University of Chicago \\
\hline Baruch Lev & New York University \\
\hline
\end{tabular}

Fonte: Elaborado pelos autores

Quadro 8 ॥ Pesquisadores da Área Contábil

\begin{tabular}{|l|c|c|}
\hline Universidade & $\begin{array}{c}\text { Número total } \\
\text { de professores }\end{array}$ & $\begin{array}{c}\text { Número de professores com } \\
\text { doutorado na própria universidade }\end{array}$ \\
\hline USP & 30 & 30 \\
\hline Pennsylvania & 16 & 0 \\
\hline Chicago & 24 & 4 \\
\hline Texas-Austin & 20 & 2 \\
\hline Illinois & 14 & 4 \\
\hline Michigan & 17 & 0 \\
\hline Stanford & 14 & 3 \\
\hline New York & 28 & 4 \\
\hline Southern California & 44 & 2 \\
\hline North Carolina & 13 & 0 \\
\hline Northwestern & 14 & 2 \\
\hline Columbia & 17 & 0 \\
\hline
\end{tabular}




\begin{tabular}{|l|c|c|}
\hline Universidade & $\begin{array}{c}\text { Número total } \\
\text { de professores }\end{array}$ & $\begin{array}{c}\text { Número de professores com } \\
\text { doutorado na própria universidade } \\
1\end{array}$ \\
\hline Indiana & 16 & 0 \\
\hline Ohio State & 26 & 2 \\
\hline Ca-Berkeley & 16 & 0 \\
\hline Cornell & 12 & 1 \\
\hline Rochester & 11 & $n . m$. \\
\hline MIT & 5 & $n . m$. \\
\hline Florida & 17 & 1 \\
\hline Washington & 9 & 1 \\
\hline Wisconsin & 16 & 3 \\
\hline Texas A\&M & 39 & 5 \\
\hline Georgia & 19 & $n . m$. \\
\hline Arizona State & 41 & 1 \\
\hline Arizona & 19 & 1 \\
\hline lowa & 20 & 2 \\
\hline Michigan State & 31 & 3 \\
\hline UCLA & 16 & $n . m$. \\
\hline
\end{tabular}

Fonte: Elaborado pelos autores

Nota: * n.m. = não mencionado

Quadro 9 Endogenia do Corpo Docente

\section{CONCLUSÕES}

Este trabalho buscou conhecer algumas das características dos principais programas de doutorado em contabilidade dos Estados Unidos. A amostra do estudo consistiu em 28 universidades que possuem um programa de doutorado em contabilidade (Ph.D) ou doutorado em administração com concentração em contabilidade (DBA). Para seleção dessas universidades, utilizou-se como base o ranking publicado pela revista de negócios U.S. News \& World Report - America's Best Graduate Schools in Accounting-2006. Com base nos resultados evidenciados, pode-se inferir algumas características a respeito dos programas, são elas:

- O processo seletivo para ingresso nos programas de doutorado analisados é baseado em dois testes: GMAT / GRE e TOEFL (para estrangeiros), nas notas dos cursos de graduação e pós-graduação dos alunos e nas recomendações sobre os estudantes. Todos os cursos analisados exigem dedicação exclusiva dos alunos de doutorado. Contrariamente, conhecimentos específicos de contabilidade, publicações e análise do pré-projeto de tese não são utilizados como critérios no processo seletivo dos programas analisados.

- Além da defesa da tese de doutorado e do cumprimento do número total de disciplinas, na maioria dos cursos analisados existe um teste oral/escrito que envolve disciplinas como contabilidade, métodos estatísticos e economia. Somente após aprovação nesse teste, o estudante torna-se um Ph.D Candidate (candidato ao Doutorado). Publicações em congresso e accounting foruns, também são exigidas e o número de artigos varia de 1 a 3 , dependendo do programa.

- O foco principal dos programas é a pesquisa (research). Isso é evidenciado através do alto número de disciplinas envolvendo metodologia científica, estatística, econometria, sociologia e psicologia. Disciplinas mais específicas de contabilidade visando à formação profissional dos estudantes (Certified Public Accountant-CPA) não são abordadas nos programas de doutorado.

- O perfil do corpo docente desses programas é altamente heterogêneo. As análises evidenciaram que, em algumas universidades, nenhum professor do departamento de contabilidade possui doutorado naquela mesma universidade.

Ressalte-se que este estudo de abordagem exploratória não teve como objetivo esgotar o tema analisado e os resultados encontrados nele não devem ser generalizados para outras amostras. A pesquisa tampouco tem por objetivo criticar o programa de doutorado em contabilidade da Universidade de São Paulo. Vale ressaltar que a USP, através do seu programa de pós-graduação em contabilidade, já formou aproximadamente 420 mestres e 150 doutores em contabilidade. Também é responsável pela publicação do único periódico de contabilidade classificado com nota "A - Nacional" na avaliação da Qualis/Capes. Além disso, os 13 mestrados em contabilidade existentes no cenário 
nacional, atualmente, são resultado do trabalho executado pela USP nos últimos 30 anos.

Entretanto, para o crescimento e evolução de uma disciplina faz-se necessário um embate de diferentes enfoques (pontos de vista) originários de diferentes formações, insti- tuições e países. Nesse sentido, o presente trabalho busca contribuir para a construção e o aperfeiçoamento da contabilidade, na medida em que evidencia possibilidades de melhorias nos programas de pós-graduação no Brasil.

\section{Referências Bibliográficas}

AMERICAN ACCOUNTING ASSOCIATION. Disponível em: <http://aaahq.org/index.cfm>.

ASSOCIATION to ADVANCE COLLEGIATE SCHOOLS of BUSINESS. Disponível em: < http://www.aacsb.edu.>.

BEATTIE, V.; GOODACRE, A.. Publishing patterns within the UK accounting and finance academic community. The British Accounting Review. Article in the press, p. 1-38, 2003.

BEDFORD, N.. A history of accountancy. Center of International Education and Research in Accounting, Champaign, IL, 1997.

BELL, T.; FRECKA, T.; SOLOMON, I.. The relation between research productivity and teaching effectiveness: empirical evidence for accounting educators. Accounting Horizons. V.7, n.4, p. 33-49, 1993.

BROWN, L.; INDRARINI, L.. Ranking accounting Ph.D. programs and faculties using social science research network downloads. Social Science Research Network - SSRN. September 23, 2003.

CARCACELLO, J. et al. Doctoral study in accounting: costs and financial support. Issues in Accounting Education. V.9, p.219-237, 1994.

CARPENTER, C.; ROBSON, G.. Declining doctoral output in accounting. The CPA Journal. P. 68-69, August 2004.

CRUM, W.. Newest survey of doctoral programs in accounting. Journal of Accountancy. P.99-104, Oct. 1974.

DOOGAR, R.. What should accounting doctoral programs focus on? An economic perspective. Advances in Accounting Education. V.5, p. 199210,2003

EVERETT, J.; KLAMM, B.; STOLTZFUS, R.. Developing benchmarks for evaluating publication records at doctoral programs in accounting. Journal of Accounting Education. V.22, p.229-252, 2004.

GABBIN, A.. The crisis in accounting education. Journal of Accountancy. P.81-85, April 2002.

GERMANO, C.. Retrospectiva das teses de contabilidade até 1988. 1988. Dissertação (Mestrado). Faculdade de Economia, Administração e Contabilidade. Universidade de São Paulo, São Paulo.

HASSELBACK, J.. Accounting faculty directory. Englewood Clifs, NJ: Prentice-Hall. 2001.

Personal webpage. Disponível em: <http://www.jrhasselback.com/Articles/>. Acesso em: 18/01/2005.

JOURNAL OF CITATION RESEARCH -JCR. Disponível em: <www.isiknowledge.com>.

JOHNSON, S.. The economic function of doctoral programs in accounting: alternative theories and educational implications. The Accounting Review. N.4, Oct. 1985.

KINNEY, W.. New accounting scholars - does it matter what we teach them? Issues in Accounting Education. Vol.18, n.1, 2003.

MARION, J.. Aspectos do ensino da contabilidade nos Estados Unidos. Caderno de Estudos Fipecafi. N.7, p.1-66, 1992.

MEYER, M.; TITARD, P.. Those who can... teach. Journal of Accountancy. V. 190, p.49-58, 2000.

MINTZBERG, H.. MBA? Não obrigado - Uma visão crítica sobre a gestão e o desenvolvimento de gerentes. Tradução Bazan Tecnologia e Linguística. Porto Alegre: Bookman, 2006.

POLICANO, A.. What price rankings? Business Education. P.26-32, sept./oct. 2005.

REINSTEIN, A.; HASSELBACK, J.. A literature review of articles accessing the productivity of accounting faculty members. Journal of Accounting Education. Vol.15, p.425-455, 1998.

RICCIO, E.; CARASTAN, J.; SAKATA, M.. Accounting research at Brazilian universities. Asian Pacific Conference. Melbourne, Australia, 1999. SHIPLEY, K.; ENGLE, R.. We nee more Ph.D.s in accounting. Management Accounting. Vol.54, n.5, p.36- 40, 1982.

SIEMENS, J. et al. An examination of the relationship between research productivity in prestigious business journal and popular press business school rankings. Journal of Business Research. V. 25, p. 467-476, 2005.

STAMMERJOHAN, W; HALL, S.. Evaluation of doctoral programs in accounting: an examination of placement. Journal of Accounting Education. Vol.20, p.1-27, 2002.

U.S. NEWS \& WORLD REPORT. Disponível em: $<$ http://www.usnews.com/usnews/rankguide $>$.

WILLIAMS, F.. Enrollment up 40\% at University of Buffalo. MBA program. NY: Buffalo News. Sept. 2001.

\section{NOTA - Endereço dos autores}

Universidade Federal de Santa Catarina

Centro Sócio-Econômico

Departamento de Ciências Contábeis
Campus Universitário - Trindade

CEP 88040-900 - Florianópolis - SC 